\title{
latrogenic aortic dissection after minimally invasive aortic valve replacement: a case report
}

\author{
Mohamed Ehab Ramadan', Lamia Buohliqah², Juan Crestanello ${ }^{3}$, James Ralston ${ }^{4}$, David Igoe ${ }^{4}$ and Hamdy Awad ${ }^{1 *}$
}

\begin{abstract}
Background: As minimally invasive cardiac and vascular procedures are on the rise, the incidence of iatrogenic acute aortic dissection (IAAD) will increase. Cardiovascular professionals should be aware about the risk factors, means of prevention and best management options for IAAD in the perioperative setting.

Case presentation: We present the successful clinical management of a complicated case of IAAD after minimally invasive aortic valve replacement.

Conclusion: High index of suspicion is required for prompt diagnosis of IAAD; collaboration of the whole perioperative team is imperative for management of this catastrophe.

Keywords: Case Report, latrogenic aortic dissection, AVR, PCI, TEVAR, TAVR

Abbreviations: ACT, Activated clotting time; APTT, Activated partial thromboplastin time; AVR, Aortic valve replacement; CPB, Cardiopulmonary bypass; ECLS, Extracorporeal life support; ECMO, Extracorporeal membrane oxygenation; ELSO, Extracorporeal life support organization; IAAD, latrogenic acute aortic dissection; NHLBI, National, heart, lung and blood institute; PCI, Percutaneous coronary intervention; ROTEM, Rotational thromboelastometry; TAVR, Transaortic valve replacement; TEE, Transesophageal echocardiography; TEVAR, Thoracic endovascular aortic repair; UNFH, Unfractionated heparin; VA ECMO, Venoarterial Extracorporeal membrane oxygenation
\end{abstract}

\section{Background}

Iatrogenic acute aortic dissection (IAAD) is a fatal complication that can occur after open cardiac surgery, complex percutaneous coronary intervention $(\mathrm{PCI})$, thoracic endovascular aortic aneurysm repair (TEVAR) and transaortic valve replacement (TAVR). As minimally invasive procedures are on the rise, the incidence of IAAD will increase. IAAD carries high mortality and represents a huge challenge to all cardiovascular professionals including cardiac surgeons who encounter this clinical scenario [1]. Here we present the successful clinical management of a case of IAAD after minimally invasive aortic valve replacement (AVR). We will review the literature for incidence, risk factors, diagnosis and management of IAAD in open cardiac surgery, PCI, TEVAR and TAVR.

\footnotetext{
* Correspondence: Hamdy.Elsayed-awad@osumc.edu

'Department of Anesthesiology, The Ohio State University, Wexner Medical Center, N411 Doan Hall, 410 West 10th Avenue, Columbus, OH 43210, USA Full list of author information is available at the end of the article
}

\section{Case presentation}

Sixty-seven years old female patient with history of atypical chest pain, exertional dyspnea, asthma and hypothyroidism was diagnosed with aortic insufficiency. Her preoperative echocardiography findings were dilated aortic root $(3.7 \mathrm{~cm})$, mild left ventricular hypertrophy and ejection fraction (70 \%). Evaluation by cardiac catheterization revealed severe aortic regurge and normal coronary arteries. An $8 \mathrm{~cm}$ median sternotomy was performed. The distal ascending aorta was cannulated at blood pressure of $100 \mathrm{mmHg}$ systolic and cardiopulmonary bypass (CPB) was initiated with good flow and pressures in the cannula. The surgeon noticed a hematoma around the cannulation site. With the aid of Transesophageal echocardiography (TEE) done by the anesthesiologist, diagnosis of a type A acute aortic dissection was made, that was extending to the aortic root and the descending aorta (Fig. 1). CPB was terminated without any significant event, and right common femoral artery was cannulated for alternate route of CPB. The 


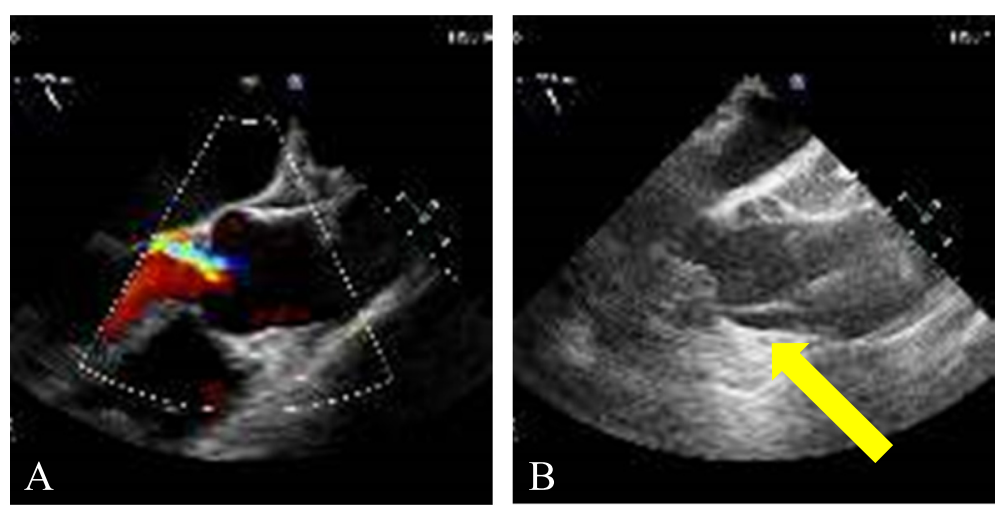

Fig. 1 Diagnosis of IAAD by TEE. TEE Mid Esophageal Ascending Aorta Long Axis view. a Before aortic cannulation; b After aortic cannulation showing intimal flap on the anterior aortic wall at the sinotubular junction with the development of true and false aortic lumens and extending antegrade and retrograde: Type A acute aortic dissection (Yellow Arrow)

sternotomy incision was extended to a full sternotomy. Cooling for $48 \mathrm{~min}$ to 18 degrees Centigrade nasopharyngeal temperature was achieved for deep hypothermic circulatory arrest. Bentall procedure with a composite Carpentier-Edwards Perimount $23 \mathrm{~mm}$ tissue valve and a $28 \mathrm{~mm}$ Hemashield graft was performed. Circulation was resumed and patient was rewarmed. Both coronary arteries buttons were attempted but failed due to frail intima at the coronary ostia. Left main coronary artery was reimplanted via a Cabrol procedure with an $8 \mathrm{~mm}$ Hemashield graft. Bypass of the right coronary artery with reverse saphenous vein graft was also done. We weaned the patient from $\mathrm{CPB}$ but there was progressive dilatation of the right ventricle and left ventricular dysfunction, so we were concerned with the graft for the left main coronary artery. We went back on CPB to perform bypasses to the left anterior descending artery and the obtuse marginal artery using reverse saphenous vein grafts. Then, we weaned the patient from $\mathrm{CPB}$ again with difficulty on high doses of inotropic support. Due to long CPB time (336 minutes), the team decided to support the patient with venoarterial extracorporeal membrane oxygenation (VA ECMO) by re-cannulating the right common femoral artery and vein. The chest was left open at the end of the procedure due to cardiac edema and distension. The patient was transferred to the ICU in extremely critical condition. The cross-clamp time was $70 \mathrm{~min}$ and circulatory arrest time was $17 \mathrm{~min}$. The patient required massive blood transfusion (140 units of blood products) in the perioperative period. The chest wall was closed 3 days postoperative and was kept on ECMO support for 17 days. The patient had a vigorous postoperative course complicated by cardiac tamponade that required multiple bedside washouts. She also suffered from subacute right middle cerebral artery territory infarct that developed intraoperative with residual left sided hemiparesis. She also had prolonged intubation due to failure of multiple spontaneous breathing trials and she required tracheostomy on the twentieth postoperative day. Her last echocardiography findings before discharge were normal right and left ventricular sizes with mild to moderate systolic dysfunction (Ejection fraction: 30-35\%). The patient was discharged on the 35th postoperative day and she is continuing her rehabilitation and following up. One year later, she is totally independent and in good health, and her echocardiography showed normal ejection fraction.

\section{Risk factors of IAAD}

IAAD after cardiac surgery is a rare and devastating complication. Here we present a case of successful management after very complicated clinical course. Cannulation of the ascending aorta for CPB was first announced in 1959 [2], and since then, IAAD continues to be a fearful fatal complication that turns routine open cardiac surgery into a major clinical challenge to the team who faces it.

Our patient had three risk factors: hypertension, aortic regurgitation and thinned dilated aorta, which we believe predisposed her to IAAD. In a recent editorial in which IAAD was described as a perfect storm, the author suggested to consider replacement of the ascending aorta in patients having these three risk factors [1].

Other risk factors for IAAD include old age, known atherosclerotic disease and previous CABG surgery [3]. In addition, high and low blood pressures during insertion of the aortic cannula play a role in the development of IAAD [4]. Very low blood pressure renders the aortic wall unsupported during cannulation with increased liability to dissection.

\section{Recent classification for IAAD in cardiac surgery}

Recently, in a multicenter study by Stanger et al. [5], IAAD following cardiac surgery is classified into intraoperative, early postoperative (symptoms of aortic dissection within 30 days of surgery with high risk of aortic rupture and intraoperative mortality) and late postoperative (after 
30 days from cardiac surgery). They further classified late postoperative IAAD into acute (patients required intervention within 2 weeks of symptoms) and chronic (late incidental finding of dissection with no acute symptoms, candidates for elective surgery) (Table 1).

\section{Incidence and common entry sites of IAAD}

Literature shows the incidence of IAAD to be 0.12 $0.23 \%$; mortality rates range from 20 to $43 \%[6,7]$, with decreased rates when IAAD is diagnosed intraoperatively, and its extension is limited to the aortic arch [8].
Most dissection sites mentioned in the literature after open cardiac surgery were related to aortic cannulation, aortic cross-clamping, aortotomy, vein anastomosis during CABG, the cardioplegia cannula, direct aortic injury and injury related to flow of the bypass to the aortic arch or descending thoracic aorta [5, 9-12]. Reviewing the literature in 3 single center and 1 multicenter studies showed nearly the same trends in sites of IAAD over that last 35 years (Table 1 ) [5, 10-12]. In the majority of cases, the site of dissection in intraoperative cases was the cannulation territory which is consistent with our case.

Table 1 Trends in sites of iatrogenic aortic dissection after cardiac surgery in literature

\begin{tabular}{|c|c|c|c|c|}
\hline & $\begin{array}{l}\text { Gott et al. [10] } \\
(N=27 / 11,145) \\
1982-1988\end{array}$ & $\begin{array}{l}\text { Still et al. [11] } \\
(N=24 / 14,877) \\
1980-1990\end{array}$ & $\begin{array}{l}\text { Leontyev et al. [12] } \\
(N=36 / 55,279) \\
1995-2010\end{array}$ & $\begin{array}{l}\text { Stanger et al. [5] } \\
(N=103 / 68,249) \\
2006-2010\end{array}$ \\
\hline \multicolumn{5}{|l|}{ 1- Intraoperative } \\
\hline & $(N=27)$ & $(N=20)$ & $(N=31)$ & $(N=24)$ \\
\hline Aortic cannulation & $10(37 \%)^{a}$ & $10(50 \%)^{a}$ & $12(38 \%)^{a}$ & $7(29.1 \%)^{a}$ \\
\hline Cardioplegia cannula & $5(18.5 \%)$ & $0(0 \%)$ & 7 (22.6 \%) & 4 (16.7 \%) \\
\hline Aortic cross-clamp & $4(14.8 \%)$ & $8(40 \%)$ & 4 (12.9\%) & $7(29.1 \%)^{a}$ \\
\hline Proximal anastomosis & $2(7.4 \%)$ & $1(5 \%)$ & $1(3.2 \%)$ & $5(20.8 \%)$ \\
\hline Direct aortic injury & $0(0 \%)$ & $1(5 \%)$ & $0(0 \%)$ & $0(0 \%)$ \\
\hline Unknown & $2(7.4 \%)$ & $0(0 \%)$ & 5 (16.1\%) & 1 (4.2\%) \\
\hline \multicolumn{5}{|l|}{ 2- Early Postoperative: } \\
\hline & & $(N=4)$ & $(N=5)$ & $(N=12)$ \\
\hline Aortic cannulation & & $3(75 \%)^{\mathrm{a}}$ & $2(40 \%)^{a}$ & $0(0 \%)$ \\
\hline Cardioplegia cannula & & $0(0 \%)$ & $0(0 \%)$ & $1(8.3 \%)$ \\
\hline Aortic cross-clamp & & $1(25 \%)$ & $0(0 \%)$ & 1 (8.3 \%) \\
\hline Proximal anastomosis & & $0(0 \%)$ & 1 (20 \%) & $8(66.7 \%)^{a}$ \\
\hline Aortotomy & & $0(0 \%)$ & $0(0 \%)$ & $2(16.7 \%)$ \\
\hline Unknown & & $0(0 \%)$ & $2(40 \%)^{a}$ & $0(0 \%)$ \\
\hline \multicolumn{5}{|l|}{ 3- Late: Postoperative } \\
\hline a) Acute & & & & $(N=44)$ \\
\hline Aortic cannulation & & & & $1(2.2 \%)$ \\
\hline Cardioplegia cannula & & & & $0(0 \%)$ \\
\hline Aortic cross-clamp & & & & $2(4.5 \%)$ \\
\hline Proximal anastomosis & & & & $10(22.7 \%)$ \\
\hline Aortotomy & & & & $24(54.5 \%)^{a}$ \\
\hline Unknown & & & & 7 (15.9 \%) \\
\hline b) Chronic & & & & $(N=23)$ \\
\hline Aortic cannulation & & & & $2(8.7 \%)$ \\
\hline Cardioplegia cannula & & & & $0(0 \%)$ \\
\hline Aortic cross-clamp & & & & $0(0 \%)$ \\
\hline Proximal anastomosis & & & & $9(39.1 \%)^{a}$ \\
\hline Aortotomy & & & & $11(47.8 \%)$ \\
\hline Unknown & & & & $1(4.3 \%)$ \\
\hline
\end{tabular}

${ }^{\mathrm{a}}$ Represents highest incidence in each category within every study 


\section{Prevention and management of IAAD Role of surgeons}

Aortic cannulation is the single most important step in cardiac surgery and prevention of IAAD is the best treatment strategy. Surgeons should be extremely cautious during aortic cannulation, clamp application and removal especially in patients with risk factors for IAAD. Cannulation purse-string sutures should be taken with deep partial or full thickness bites. Surgeons should also avoid torsion and proximity of the partial-occlusion clamp to the aortic cannula [13].

IAAD diagnosis requires high index of suspicion and it depends primarily on visualization and imaging. Intraoperative diagnosis is achieved by visualization of a bluish discoloration and dilatation of the aorta [9]. Still et al. [11] differentiated a subadventitial hematoma from IAAD; the subadventitial hematoma is usually small, enlarges slowly, stops enlarging after initiation of bypass, soft to palpation and can be decompressed by incising the aortic surface. IAAD, on the other hand, may involve the entire aorta or part of it, giving it a tense, dilated bluish discoloration, and bleeds rapidly on incision of the adventitial surface. Once IAAD is confirmed, the surgeon has to secure an alternative peripheral site of cannulation and ensures that the blood flow is through the true aortic lumen.

\section{Role of anesthesiologists}

Anesthesiologists are encouraged to routinely examine the cannulation site after insertion and removal of the aortic cannula as well as the other previously reported possible entry sites of dissection whenever possible using TEE. Routine use of TEE aids in the rapid diagnosis and the prompt management of IAAD with a sensitivity of $98 \%$ and specificity of $95 \%$. TEE also differentiates dissection from subadventitial hematoma. However, visualization of IAAD by TEE in the distal ascending aorta and proximal arch may be limited due to the interposition of tracheal air between the ultrasonic probe and the aorta, and may require an epiaortic echocardiographic probe $[6,14,15]$. Moreover, epiaortic scanning can be used when there is a contraindication for the use of TEE [16]. Once IAAD is confirmed and until an alternate cannulation site is secured, the anesthesiologists have an important role to support the circulation during the transition from $\mathrm{CPB}$ to spontaneous circulation in order to maintain critical organs perfusion.

\section{Role of perfusionist}

Communication between the perfusionist and the surgeon is mandatory during the test transfusion to ensure proper placement of the aortic cannula. The pump should be started slowly, while the arterial line pressure is being checked for any pressure rise denoting obstruction. Aortic dissection is among one of the causes of abrupt increase in the arterial pressure line and needs to be excluded. It is advisable for the perfusionist to reduce the pump flow $(0.5-1 \mathrm{~L} / \mathrm{min})$ during insertion or removal of the aortic cannula [17].

After diagnosis of IAAD, the underlying cause should be corrected and the management plan should be changed aiming at repair of the dissection. In cases with small intimal tear, simple primary repair could be achieved [18]. In our case, we had to initiate $\mathrm{CPB}$ through peripheral cannulation and performed hypothermic circulatory arrest for repair of the dissection. The rate of institution of hypothermic circulatory arrest for repair of IAAD mentioned in the literature ranged 50-95 \% [5, 10-12].

Ruchat et al. [9] mentioned that cerebral malperfusion is one of the unfavorable outcomes of IAAD that may be due to extension of the dissection beyond the aortic arch. In their study, two of seven patients who experienced IAAD died of hypoxic brain insult. Prompt intraoperative diagnosis and management of IAAD may prevent this sequel. In another study, stroke was reported in 27 of 103 patients with IAAD [5]. Our patient developed right middle cerebral artery infarction that was most probably due to cerebral hypoperfusion from severe hemodynamic instability or embolism, but was not explained to be due to vascular dissection neither by intraoperative evidence through direct visualization of the arch vessels or cerebral monitoring, nor by postoperative imaging using carotid Doppler and MRA.

Our patient had a complicated postoperative course in the ICU, including bleeding and recurrent cardiac tamponade. After one year follow up, our patient, is in good health owing to the team approach.

\section{ECMO support and coagulation management}

The use of ECMO as a salvage modality for cardiogenic shock, specifically post-cardiotomy cardiogenic shock, may offer advantages over conventional medical therapies $[19,20]$. The benefit of mechanical support providing improved cardiac output without the negative hypoperfusion consequences, led to the proactive initiation of VA ECMO support. Squiers et al. [21] concluded that VA ECMO offers certain advantage in rare instances of postcardiotomy shock, in addition to other studies that showed excellent long-term survival in patients surviving the acute perioperative period.

Our patient required transfusion of 140 units of blood and blood products in a very short period of time. Massively transfused patients have improved survival when receiving a high fresh frozen plasma:RBC and platelet:RBC ratio [22]. As such, we utilize point of care testing rotational thromboelastometry (ROTEM) perioperative in our patients, which leads to an increase in fresh frozen plasma and platelet to $\mathrm{RBC}$ transfusion ratio, that is consequently associated with improved survival [22]. 
Our center utilizes Extracorporeal Life Support Organization (ELSO) guidelines for anticoagulation and therapeutic management of unfractionated heparin (UNFH) during mechanical support. [20] We utilize an ECMO circuit with a biocompatible coated surface that provides a significant benefit in patients with massive coagulopathic bleeding, as it allows the delay of anticoagulation at the start of extracorporeal life support (ECLS).

The activated clotting time (ACT) is a universally accepted, and a readily available intraoperative point of care test to assess and dose UNFH. However, postoperative therapeutic anticoagulation, will be influenced by many factors including urine output, renal replacement therapy, platelet administration, and underlying coagulation changes. Additionally, the ACT test will be affected by postoperative factors such as thrombocytopenia, and smaller percent changes in individual clotting cascade factors than the activated partial thromboplastin time (APTT). A recent study in pediatric ECLS patients showed that the APTT (clinical laboratory and POC) correlated to UNFH dose better than ACT and, as expected the APTT and UNFH dose correlation improved with increasing patient age [23]. As recommended per ELSO guidelines, using one method of UNFH activity alone is considered insufficient today, and yet an array of too many tests for UNFH therapy, multiple times per day, could provide possible confusion to ECLS team members [20]. Our center therefore monitors UNFH activity during the postoperative course with the more sensitive APTT test, to maintain a therapeutic level, and supplementation of ROTEM tests as needed.

IAAD in other minimally invasive cardiovascular procedures IAAD can present with other cardiovascular procedures: during diagnostic and therapeutic coronary artery intervention, TEVAR and TAVR. The clinical presentation and the management vary widely.

\section{$I A A D$ during coronary artery intervention}

In the Catheterization Lab, IAAD may occur during diagnostic and therapeutic PCI. The incidence of IAAD in cardiac catheterization is less than $0.1 \%$. It may involve the coronary arteries and/or the aorta. National, Heart, Lung and Blood Institute (NHLBI) classified coronary artery dissection to 6 types, none of which involved the aortic root [24]. A simplified classification for this type of dissection was mentioned by Eshtehardi et al. [25]: type $I$ (localized dissection in the coronary arteries), type II (extension of dissection into major coronary branches), and type III (extension of the dissection into the aortic root). Cardiac surgeons will manage emergency cardiac surgeries in a small fraction of cases of cardiac catheterization complicated by IAAD. Current data on incidence and the clinical outcome of IAAD in cardiac catheterization is limited. In multicenter study the incidence of IAAD was $0.062 \%$, of those involving the coronary tree was $0.039 \%$ with higher incidence in therapeutic than in diagnostic procedures. The operator expertise and the use of guiding wires in complex procedures are suggested risk factors leading to IAAD after PCI [26].

In a study by Leontyev et al. [12], 12 out of 135,262 patients developed IAAD after cardiac catheterization; all patients underwent surgical intervention with 30 day mortality of $50 \%$ denoting high mortality in this group of patients. In another registry, only 3 patients out of 74 patients with IAAD after PCI were referred to surgery. The rest of the patients were managed either conservatively or by PCI [26]. This might be attributed to difference in local practice and/or the type and extent of dissection. In patients with retrograde dissection not involving the coronary arteries, conservative management may be attempted together with close patient monitoring for possible surgical intervention. On the other hand, antegrade dissections usually require endovascular or surgical management [26].

\section{IAAD during TEVAR}

Minimal invasive techniques have changed clinical practice for management of thoracic aortic diseases. TEVAR is the first choice therapy for most thoracic aortic disease. IAAD after TEVAR was also reported in the literature with incidence ranging 1-6.8 \% [27, 28]. Several factors leading to this complication have been proposed: instrumentation and stent negotiation, underlying aortic pathology (e.g. aneurysm, ulcer) leading to fragile wall, oversized grafts, excessive aortic arch angulation and expanding intramural hematoma $[29,30]$. Even with immediate open surgical management to prevent grave outcomes, mortality rate in the literature is $44-57 \%$ [30].

\section{IAAD during TAVR}

With the rapid increase in the number of TAVR cases around the globe, the association between IAAD and TAVR is also emerging in the literature. IAAD after TAVR procedure is mentioned as part of the major vascular complications. The incidence ranges from $0.2 \%$ in the GARY registry (33 IAAD of 15,964 patients) to $2 \%$ in transapical TAVR cases in another review [31, 32]. In the PARTNER trial, there were 3 patients with IAAD out of 419 included in the trial; however, in the three studies, specific clinical management of IAAD was not mentioned [33].

\section{Conclusion}

IAAD is a dreadful complication that carries high morbidity and mortality. All cardiovascular professionals should be ready to diagnose and quickly intervene specially with the increase in numbers and complexity of minimally invasive procedures. 


\section{Funding}

The authors have received no funding for this work.

\section{Availability of data and materials}

Not Applicable.

\section{Authors' contributions}

HA provided anesthetic management for the case, and helped with the drafting and proofreading of the manuscript. MER, LB, JR and DI participated in background literature review, drafting, and proofreading of the manuscript. $\mathrm{JC}$ is the lead surgeon on the case; he emphasized the significance of this topic and helped proofread the manuscript. JR and DI are from the perfusionist team at our institution who participated in the perioperative management of this case. All authors read and approved the final manuscript.

\section{Competing interests}

The authors declare that they have no competing interests.

\section{Consent for publication}

Written informed consent was obtained from the patient for publication of this Case report and any accompanying images. A copy of the written consent is available for review by the Editor-in-Chief of this journal.

\section{Author details}

${ }^{1}$ Department of Anesthesiology, The Ohio State University, Wexner Medical Center, N411 Doan Hall, 410 West 10th Avenue, Columbus, OH 43210, USA. ${ }^{2}$ Department of Otolaryngology - Head \& Neck Surgery, The Ohio State University, Wexner Medical Center, Columbus, OH, USA. ${ }^{3}$ Department of Surgery, Division of Cardiac Surgery, The Ohio State University, Wexner Medical Center, Columbus, OH, USA. ${ }^{2}$ Perfusion Services, The Ohio State University, Wexner Medical Center, Columbus, OH, USA

Received: 16 May 2016 Accepted: 23 August 2016

Published online: 24 August 2016

\section{References}

1. Cleveland Jr JC. A perfect storm: type A aortic dissection and previous cardiac surgery. Circulation. 2013;128(15):1593-4.

2. Nunez LE, Bailey CP. New method for systemic arterial perfusion in extracorporeal circulation. J Thorac Surg. 1959:37(6):707-10.

3. von Kodolitsch $Y$, Simic O, Schwartz A, Dresler C, Loose R, Staudt M, et al. Predictors of proximal aortic dissection at the time of aortic valve replacement. Circulation. 1999:100(19 Suppl):II287-294.

4. Gharde P, Aggarwal V, Chauhan S, Kiran U, Devagourou V. latrogenic acute aortic dissection during cardioplegic cannula insertion detected by transesophageal echocardiography. J Cardiothorac Vasc Anesth. 2012;26(1):e3-5.

5. Stanger O, Schachner T, Gahl B, Oberwalder P, Englberger L, Thalmann M, et al. Type A aortic dissection after nonaortic cardiac surgery. Circulation. 2013; 128(15):1602-11.

6. Fleck T, Ehrlich M, Czerny M, Wolner E, Grabenwoger M, Grimm M. Intraoperative iatrogenic type A aortic dissection and perioperative outcome. Interact Cardiovasc Thorac Surg. 2006;5(1):11-4.

7. Hurt A, Smith JM, Engel AM. Predictors and outcomes associated with intraoperative aortic dissection in cardiac surgery. J Card Surg. 2008;23(5):422-5.

8. Hwang HY, Jeong DS, Kim KH, Kim KB, Ahn H. latrogenic type A aortic dissection during cardiac surgery. Interact Cardiovasc Thorac Surg. 2010;10(6):896-9.

9. Ruchat P, Hurni M, Stumpe F, Fischer AP, von Segesser LK. Acute ascending aortic dissection complicating open heart surgery: cerebral perfusion defines the outcome. Eur J Cardiothorac Surg. 1998;14(5):449-52.

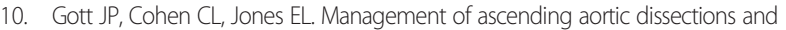
aneurysms early and late following cardiac operations. J Card Surg. 1990:5(1):2-13.

11. Still RJ, Hilgenberg AD, Akins CW, Daggett WM, Buckley MJ. Intraoperative aortic dissection. Ann Thorac Surg. 1992;53(3):374-9. discussion 380.

12. Leontyev S, Borger MA, Legare JF, Merk D, Hahn J, Seeburger J, et al. latrogenic type A aortic dissection during cardiac procedures: early and late outcome in 48 patients. Eur J Cardiothorac Surg. 2012:41(3):641-6.

13. Singh A, Mehta Y Intraoperative aortic dissection. Ann Card Anaesth. 2015;18(4):537-42.
14. Assaad S, Geirsson A, Rousou L, Sherman B, Perrino A. The dual modality use of epiaortic ultrasound and transesophageal echocardiography in the diagnosis of intraoperative iatrogenic type-a aortic dissection. J Cardiothorac Vasc Anesth. 2013;27(2):326-8.

15. Shanewise JS, Cheung AT, Aronson S, Stewart WJ, Weiss RL, Mark JB, et al. ASE/SCA guidelines for performing a comprehensive intraoperative multiplane transesophageal echocardiography examination: recommendations of the American Society of Echocardiography Council for Intraoperative Echocardiography and the Society of Cardiovascular Anesthesiologists Task Force for Certification in Perioperative Transesophageal Echocardiography. Anesth Analg. 1999:89(4):870-84.

16. Jansen Klomp WW, Brandon Bravo Bruinsma GJ, van 't Hof AW, Grandjean JG, Nierich AP. Imaging Techniques for Diagnosis of Thoracic Aortic Atherosclerosis. Int J Vasc Med. 2016;2016:4726094.

17. Ghosh S, Falter F, Cook DJ. Cardiopulmonary bypass. Cambridge: Cambridge University Press; 2009

18. Varghese D, Riedel BJ, Fletcher SN, Al-Momatten MI, Khaghani A. Successful repair of intraoperative aortic dissection detected by transesophageal echocardiography. Ann Thorac Surg. 2002;73(3):953-5.

19. Abrams D, Combes A, Brodie D. Extracorporeal membrane oxygenation in cardiopulmonary disease in adults. J Am Coll Cardiol. 2014;63(25 Pt A):2769-78.

20. ELSO Guidelines [https://www.elso.org/Resources/Guidelines.aspx]Last Accessed 24 July 2016

21. Squiers JJ, Lima B, DiMaio JM. Contemporary extracorporeal membrane oxygenation therapy in adults: Fundamental principles and systematic review of the evidence. J Thorac Cardiovasc Surg. 2016;152(1):20-32.

22. Johansson Pl. Hemostatic strategies for minimizing mortality in surgery with major blood loss. Curr Opin Hematol. 2009;16(6):509-14.

23. Maul TM, Wolff EL, Kuch BA, Rosendorff A, Morell VO, Wearden PD. Activated partial thromboplastin time is a better trending tool in pediatric extracorporeal membrane oxygenation. Pediatr Crit Care Med. 2012;13(6):e363-371.

24. Rogers JH, Lasala JM. Coronary artery dissection and perforation complicating percutaneous coronary intervention. J Invasive Cardiol. 2004:16(9):493-9.

25. Eshtehardi $P$, Adorjan $P$, Togni $M$, Tevaearai $H$, Vogel $R$, Seiler $C$, et al. latrogenic left main coronary artery dissection: incidence, classification, management, and long-term follow-up. Am Heart J. 2010;159(6):1147-53.

26. Nunez-Gil IJ, Bautista D, Cerrato E, Salinas P, Varbella F, Omede P, et al. Incidence, Management, and Immediate- and Long-Term Outcomes After latrogenic Aortic Dissection During Diagnostic or Interventional Coronary Procedures. Circulation. 2015;131(24):2114-9.

27. Langer S, Mommertz G, Koeppel TA, Schurink GW, Autschbach R, Jacobs MJ. Surgical correction of failed thoracic endovascular aortic repair. J Vasc Surg. 2008:47(6):1195-202.

28. Neuhauser B, Czermak BV, Fish J, Perkmann R, Jaschke W, Chemelli A, et al, Type A dissection following endovascular thoracic aortic stent-graft repair. J Endovasc Ther. 2005;12(1):74-81.

29. Dong ZH, Fu WG, Wang YQ, Guo DQ, Xu X, Ji Y, et al. Retrograde type A aortic dissection after endovascular stent graft placement for treatment of type B dissection. Circulation. 2009;119(5):735-41.

30. Piffaretti G, Mariscalco G, Tozzi M, Bruno VD, Sala A, Castelli P. Acute iatrogenic type A aortic dissection following thoracic aortic endografting. J Vasc Surg. 2010:51(4):993-9.

31. Walther T, Hamm CW, Schuler G, Berkowitsch A, Kotting J, Mangner N, et al. Perioperative Results and Complications in 15,964 Transcatheter Aortic Valve Replacements: Prospective Data From the GARY Registry. J Am Coll Cardiol. 2015;65(20):2173-80

32. von Segesser LK, Gerosa G, Borger MA, Ferrari E. Prevention and management of potential adverse events during transapical aortic valve replacement. J Heart Valve Dis. 2013:22(3):276-86.

33. Genereux P, Webb JG, Svensson LG, Kodali SK, Satler LF, Fearon WF, et al. Vascular complications after transcatheter aortic valve replacement: insights from the PARTNER (Placement of AoRTic TraNscathetER Valve) trial. J Am Coll Cardiol. 2012;60(12):1043-52. 